\title{
Medication-Related Osteonecrosis of the Jaw by Concomitant Nivolumab and Denosumab Treatment
}

\author{
Gianfilippo Nifosi $^{1^{*}}$, Lorenzo Nifosi ${ }^{2}$ and Antonio Fabrizio Nifosi ${ }^{3}$ \\ ${ }^{1}$ Head of Oncology and Hematology Departement, Cancer Unit of VSHA/Alia Foundation, Sallanches, France \\ ${ }^{2}$ Dental Surgeon, Santéa group, Paris, France \\ ${ }^{3}$ Dental Surgeon, San Donato group, Milan, Italy
}

*Corresponding author: Gianfilippo Nifosì, Tel: +33772313243

\begin{abstract}
Medication-related osteonecrosis of the jaw is a multifactorial disease described in association with antiresorptive drugs, such as bisphosphonates and denosumab. More recently, cases due to non-antiresorptive medications, particularly, some cases by immune checkpoint inhibitors, have also been reported. Dentists and oral surgeons in the coming years will see an increasing number of patients who are receiving treatment potentially toxic to bone. We reported a case of a cancer patient treated with denosumab that presented MRONJ following treatment with nivolumab, with the onset of symptoms after three months (four doses) of drug. According revised literature, the physiopathological mechanisms, clinical and therapeutic aspects are discussed.
\end{abstract}

\section{Introduction}

Medication-related osteonecrosis of the jaw (MRONJ) is a well know uncommon complication by antiresorptive drugs defined by three features: 1) current or previous treatment with antiresorptive drugs; 2 ) exposed bone or bone that can be probed through an intraoral or extraoral fistula in the maxillofacial region that stays for longer than 8 weeks, and 3) no previous history of radiation therapy or obvious metastatic disease [1]. It affects the patient's quality of life, its pathogenesis is still unclear and for which there is no standard treatment. In recent years, the number of MRONJ cases has been constantly increasing due to new molecular target and immunological drugs introduced for cancer treatment and that have no antiresorptive properties. Among these, we have already reported the association of MRONJ and Tyrosine Kinase Inhibitors (TKIs),
Mammalian Target of Rapamycin Inhibitors (mTOR Inhibitors), Anti-angiogenics and Immune Checkpoint Inhibitors (ICI) [2]. Particularly, given the increasing use in cancer treatment, in recent years reports increased of MRONJ-ICI related, used alone or in combination.

\section{Case Description}

A 67-year-old woman with metastatic head and neck cancer diagnosed in March 2019, was referred in the department of odontostomatological sciences in September 2020 for pain and gingival swelling. Clinical history was positive for treated hyperfunctioning multinodular thyroid goiter and smoking (38 packed years) quit at the time of cancer diagnosis. She had been diagnosed with stage IV squamous cell carcinoma at undetermined site of onset but that it was widespread metastases to the cervical lymph nodes, lung and bone. Treatment with palliative antalgic radiotherapy on the shoulder and first line of palliative chemotherapy according to Extreme protocol (six cures of cisplatin, 5 fluorouracil and cetuximab) achieving a partial response without serious adverse events. After dental evaluation, she had been treat with denosumab $120 \mathrm{mg}$ subcutaneously every four weeks starting from September 2019. In April 2020 new hospitalization for pain in the pelvis and lower limbs, degradation of the general state, weight loss and anorexia. A computed tomography (CT) showed osteolytic lesions spread to the spine and pelvis, cervical and mediastinal lymph nodes, lung and liver metastasis. After careful endocrinological evaluation, second-line treatment with Nivolumab $240 \mathrm{mg}$ fixed 
dose every two weeks was started in June 2020 and Denosumab was continued. After receiving four doses of Nivolumab, she start to presents pain and gingival swelling in the left retromolar areas of the mandible. Intraoral examination detected bone exposure $2.5 \times 1$ $\mathrm{cm}$ posteriorly to the included third left molar according with diagnosis of stage II ONJ. A dental panoramic radiography showed a radiolucent area with poorly defined edges in left ascending branch of the mandible (Figure 1). An incisal biopsy was performed, and histopathological findings revealed inflammatory lesions without neoplastic cells. Nivolumab and Denosumab were stopped. Conservative treatment with lincomycin $500 \mathrm{mg}$ every six hours and $2 \%$ chlorhexidine alternately with $3 \%$ hydrogen peroxide mouthwashes was started and continued for four weeks with partial resolution after six weeks. New cancer staging with PET/CT in October 2020 showed disease progression with new multiple bone, hepatic and pulmonary metastases. Due to rapidly evolving metastatic disease, no oral surgical treatment was proposed. She continued with mouth rinses and received morphine to treated cancer pain.

\section{Discussion}

The checkpoints of the immune system are receptors involved in modulating of activation of immune cells in order to limit the duration and intensity of immune reactions. These pathways are crucial for self-tolerance. Cancer cells sometimes find ways to use checkpoint pathways to avoid attacks from the immune system. Therefore, disabling immune checkpoints would help re-engage the body's immune system to fight against cancers.

Starting from this concept, the immune checkpoint inhibitors (ICI) have been developed for cancer care. Currently, the ICl which are used in cancerology target two inhibitory receptors expressed by CD8 cytotoxic $T$ lymphocytes, CD4 T helper and CD4 CD25 Foxp3 Tregulators. Cytotoxic T-Lymphocyte-Associated protein 4 (CTLA-4) intervenes early in the activation of T lym-

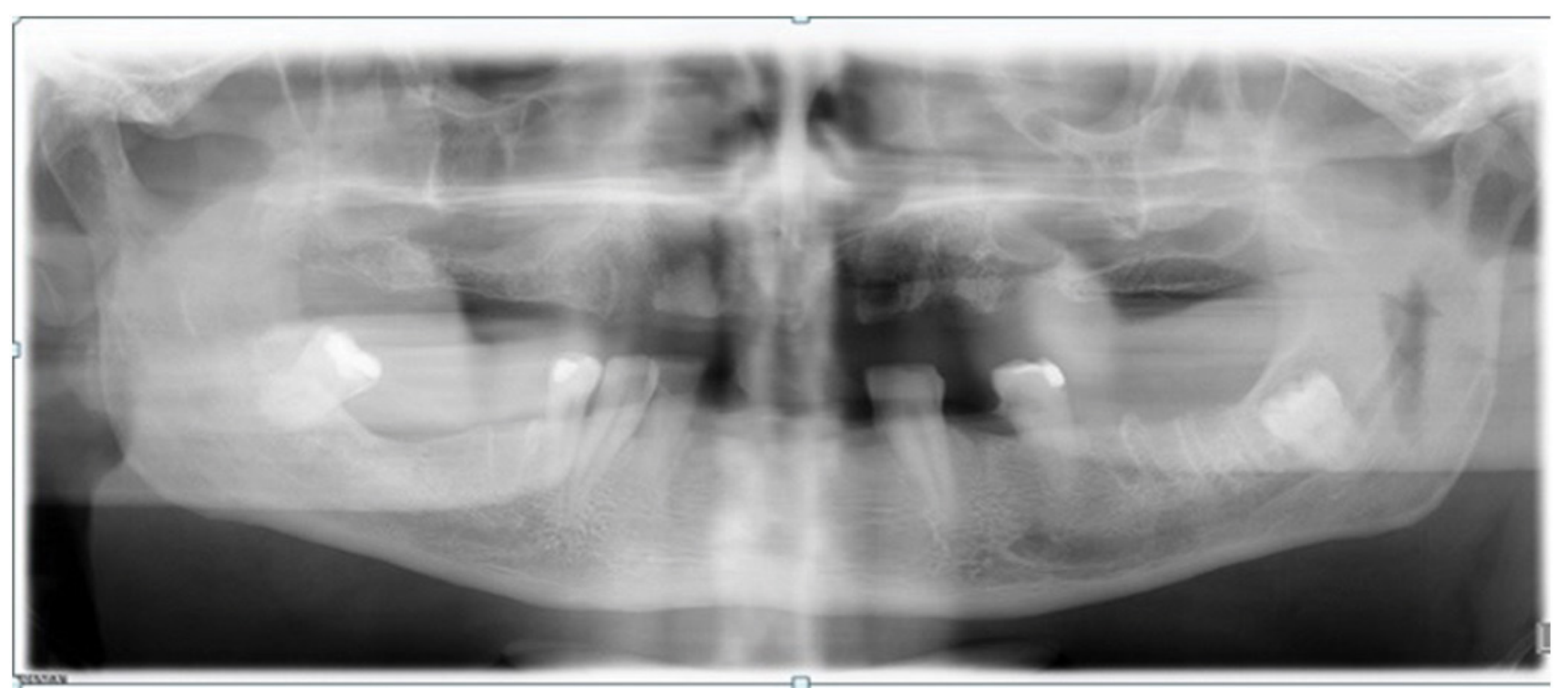

Figure 1: Radiolucent area with poorly defined edges in left ascending branch of the mandible.

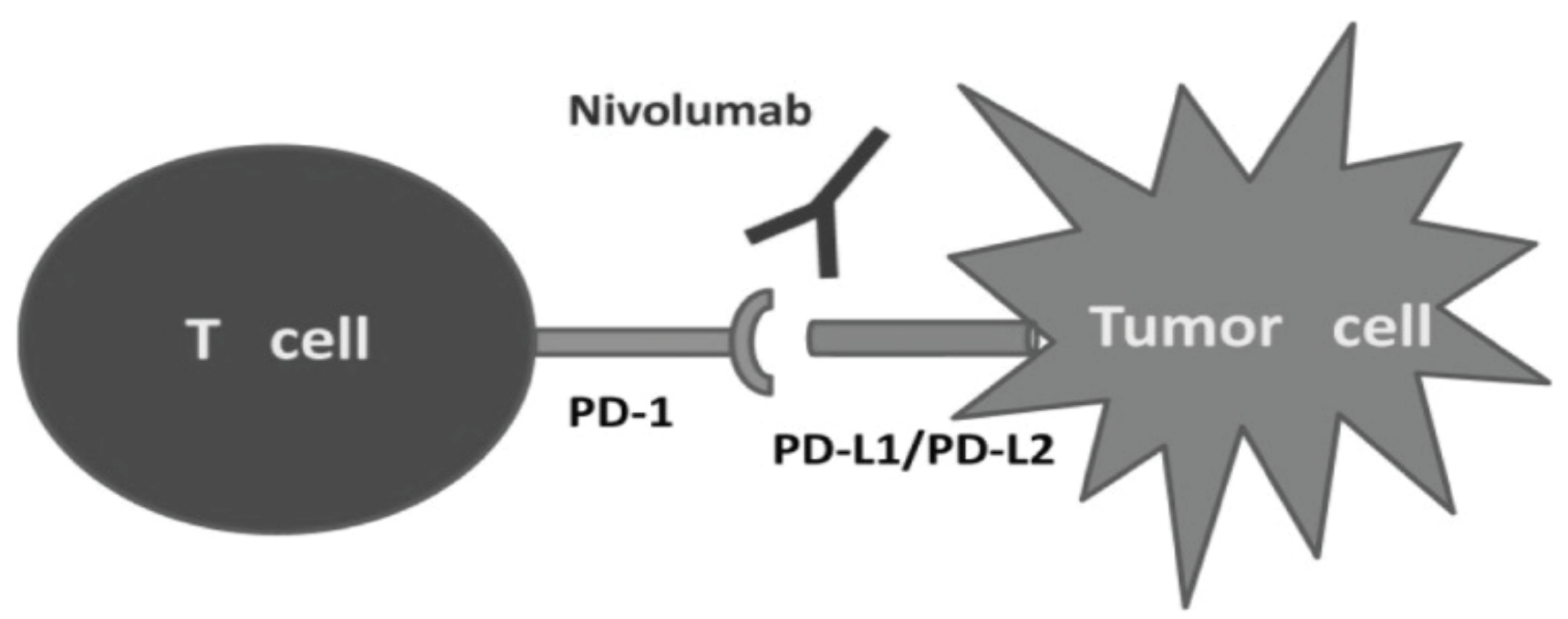

Figure 2: Schematic Nivolumab mechanism of action. 
phocytes in secondary lymphoid organs, at the time of tumor antigen presentation and binding to CD80 and CD86 inhibits T lymphocytes. The Programmed Cell Death Protein 1 (PD-1) acts later in the activation process of T lymphocytes, at the level of peripheral tissues and the tumor microenvironment, and binding its two ligands PD-L1 and PD-L2 at the surface of the cancer cells blocks the immune response. PD-L1 is highly selectively expressed on tumor infiltrating lymphocytes (TILs) from many tumors (Figure 2). The use of ICI must be able to block the functioning of these receptors thus prevent them from inhibiting the antitumor immune response. $\mathrm{ICl}$ used in the treatment of cancer are shown in Table 1.

Nivolumab is a fully human immunoglobulin G4 monoclonal antibody target to the PD1 commonly used, alone or in combination, in the treatment of many cancer and have dramatically improved survival rates in patients with metastatic cancer. It binds PD-1 with high affinity and PD-1 occupancy appeared to be dose-independent, with a mean peak occupancy of $85 \%$ at 4-24 hours. The serum half-life $\left(t_{1 / 2}\right)$ at the doses currently used is 12 days $(3 \mathrm{mg} / \mathrm{Kg}$ ) to 20 days $(10.0 \mathrm{mg} / \mathrm{kg}$ ) [3]. The safety profile of Nivolumab treatment is generally well. Like all ICl, the adverse toxicities of Nivolumab have been attributed to increased immune-reactivity against normal tissues. This immune-related adverse events (IRae) are more frequently pneumonitis or interstitial lung disease, colitis, hepatitis, nephritis, thyroid disorders, cutaneous and other immune disorders. As for the oral cavity, Nivolumab causes xerostomia

Table 1: Classification of ICI.

\begin{tabular}{|c|c|c|c|}
\hline Target & Molecule & Indication & Administration \\
\hline CTLA4 & Ipilimumab & $\begin{array}{l}\text { Melanoma } \\
\text { RCC } \\
\text { NSCLC } \\
\text { HCC } \\
\text { CRC (MSI-H) }\end{array}$ & $\begin{array}{l}\text { Intravenous, } 1 \text { infusion every } 3 \text { weeks, } 4 \\
\text { injections total }\end{array}$ \\
\hline CTLA4 & Tremelimumab & $\begin{array}{l}\text { Melanoma } \\
\text { Mesothelioma }\end{array}$ & Intravenous, 1 infusion every 3 weeks \\
\hline PD1 & Nivolumab & $\begin{array}{l}\text { Melanoma } \\
\text { NSCLC } \\
\text { SCLC } \\
\text { RCC } \\
\text { LH } \\
\text { Squamous Head \& Neck cancer } \\
\text { Urothelial cancer } \\
\text { HCC } \\
\text { CRC (MSI-H) } \\
\text { Esophageal Squamous cell cancer } \\
\text { Mesothelioma } \\
\text { TNBC }\end{array}$ & $\begin{array}{l}\text { Intravenous, } 1 \text { infusion every } 2 \text { or } 4 \\
\text { weeks }\end{array}$ \\
\hline PD1 & Pembrolizumab & $\begin{array}{l}\text { Melanoma } \\
\text { NSCLC } \\
\text { SCLC } \\
\text { LH } \\
\text { Primari mediastinal Large B-cell lymphoma } \\
\text { Head \& Neck Squamous Cell Carcinoma } \\
\text { Urothelial cancer } \\
\text { Esophageal cancer } \\
\text { Gastric cancer } \\
\text { CRC (MSI-H) } \\
\text { HCC } \\
\text { Merkel cell carcinoma } \\
\text { Cervical cancer } \\
\text { Endometrial cancer } \\
\text { RCC } \\
\text { Cutaneous squamous cell carcinoma }\end{array}$ & Intravenous, 1 infusion every 3 weeks \\
\hline PD1 & Cemiplimab & Cutaneous Squamous Cell Carcinoma & Intravenous, 1 infusion every 3 weeks \\
\hline
\end{tabular}




\begin{tabular}{|c|c|c|c|}
\hline PD-L1 & Atezolizumab & $\begin{array}{l}\text { NSCLC } \\
\text { Urothelial carcinoma } \\
\text { TNBC } \\
\text { SCLC } \\
\text { HCC } \\
\text { Melanoma }\end{array}$ & Intravenous, 1 infusion every 3 weeks \\
\hline PD-L1 & Avelumab & $\begin{array}{l}\text { Merkel cell carcinoma } \\
\text { Urothelial carcinoma } \\
\text { RCC }\end{array}$ & Intravenous, 1 infusion every 2 weeks \\
\hline PD-L1 & Durvalumab & $\begin{array}{l}\text { NSCLC } \\
\text { SCLC } \\
\text { Urothelial carcinoma }\end{array}$ & Intravenous, 1 infusion every 2 weeks \\
\hline
\end{tabular}

CRC MSI-H: Colorectal cancer microsatellite instability-high; HCC: Hepatocellular carcinoma; HL: Hodgkin lymphoma; NSCLC: Non small cell lung cancer; RCC: Renal cell carcinoma; SCLC: Small cell lung cancer; TNBC: Triple negative breast cancer.

grade $1-2$ in $6 \%$ and grade 3 exceptionally, moderate dysgeusia grade $1-2$ in less than $3 \%$ and lichenoid reactions rarely in the treated patients [4]. Some ONJ cases have been reported in recently years with $\mathrm{ICl}$ use such as Ipilimumab [5], Pembrolizumab [6] and Nivolumab [7]. Pundole, et al. examining Adverse Events Reporting System of the US Food and Drug Administration (FAERS), reported in a sample of 63.979 cancer patients with $\mathrm{ICl}$-associated adverse events between 2011 and 2018, a total of 35 ONJ cases by Nivolumab (RR 1.71, IC 0.79) and 8 by Atezolizumab (RR 2.24, IC 1.16) [8]. In a recent systematic review of the literature between 2009 and 2017 by the Bone Study Group of MASCC/ISOO, in a total of 42 ONJ cases related to non-antiresorptive drugs, a case of Nivolumab certainly related ONJ was identified out (2.3\%) [9]. The problem of MRONJ is that the cancer patients are often treated simultaneously with treatment potentially able to determine ONJ, such as chemotherapy, radiotherapy and steroids. Moreover, they often coexist key local risk factors for the development of ONJ, such as smoking, poor oral hygiene, xerostomia, dental/periodontal infections, and dentoalvelolar surgery. ICl-related ONJ have some common elements to bone targeting agents-related ONJ, such as: no gender predilection, middle-aged adults, stage $2 \mathrm{ONJ}$, mandible predilection, radiological features, symptoms, histology, and most common conservative management. Differences occurs in shorter time to onset of osteonecrosis and better prognosis.

In our case, at least two risk factors were present: smoking and Denosumab treatment. Denosumab prevents the binding of RANKL to RANK and inhibits the formation, function, and survival of osteoclasts as an anti-osteoclastogenic agent. We believe that Denosumab may have induced a subclinical ONJ whose development may have been accelerated and facilitated by Nivolumab treatment. Activation of $\mathrm{T}$ cells by this drug Nivolumab usually induces proinflammatory/pro-osteoclastogenic cytokines (IL-1, IL-6, IL-7, IL-11, IL-15, IL-17, TNF- $\alpha$, TNF- $\beta$, RANKL, and M-CSF) as well as anti-inflammatory/anti-osteoclastogenic cytokines (IL-4, IL-5, IL-10,
IL-12, IL-13, IL-18, IFN- $\gamma$, TGF- $\beta$, and OPG). Occurrence of osteonecrosis could be a consequence of the activation of a subset of anti-osteoclastogenic T lymphocites (TH1, TH2, Treg, T $\gamma \delta$ ) and NK cells, whith up regulation of IFN- $\gamma$ that inhibits osteoclastogenesis by promoting TRAF6 degradation in RANKL-induced osteoclast differentiation [10]. Finally, xerostomia can be an important local factor in the development of ONJ following immunotherapy.

\section{Conclusions}

In recent years, the link between the immune system and bone metabolism became very tight and ONJ identifies with an inflammation-associated process. The immune checkpoint inhibitors, alone or together with other factors, represent a new potential cause of ONJ's development. Risk factors must be researched and eliminated before using them in the treatment of cancer a close collaboration between the oncologist and dentist is essential for prevention, early diagnosis and treatment. Further studies are needed to elucidate the pathogenesis of $\mathrm{ICl}$-related ONJ to develop prevention strategies and rational treatment.

\section{Statement of Equal Authors' Contribution}

Gianfilippo Nifosì : observation of the case, revising it critically for important intellectual content, final approval of the version to be published.

Lorenzo Nifosì and Antonio Fabrizio Nifosì: substantial contributions to conception and design, acquisition of data, analysis and interpretation of data, drafting the article and revising.

\section{References}

1. Ruggiero SL, Dodson TB, Fantasia J, Gooday J, Aghaloo T, et al. (2014) American Association of Oral Maxillofacial Surgeons position paper on medication-related osteonecrosis of the jaw-2014 update. J Oral Maxillofac Surg 72: 1938-1956.

2. Nifosì AF, Zuccarello M, Nifosì L, Hervas Saus V, Nifosì G (2019) Osteonecrosis of the jaw in the era of targeted therapy and immunotherapy in oncology. J Korean Assoc Oral 
Maxillofac Surg 45: 3-8.

3. Guo L, Zhang H, Chen B (2017) Nivolumab as Programmed Death-1 (PD-1) ihibitor for targeted immunotherapy in tumor. J Cancer 8: 410-416.

4. Vigarios E, Epstein JB, Sibaud V (2017) Oral mucosal changes induced by anticancer targeted terapie and immune checkpoint inhibitors. Support Care Cancer 25: 17131739.

5. Owosho AA, Scordo M, Yom SK, Randazzo J, Chapman PB, et al. (2015) Osteonecrosis of the jaw a new complication related to Ipilimumab. Oral Oncol 51: e100-e101.

6. Decaux J, Magremanne M (2020) Medication-related osteonecrosis of the jaw relatd to epacadostat and pembrolizumab. J Stomatol Oral Maxillofac Surgery 121: 740-742.

7. Pundole $\mathrm{X}$, Jones $\mathrm{AL}$, Tetzlaff M, Williams MD, Murphy jr
WA, et al. (2020) Osteonecrosis of the jaw induced by treatment with anti-PD-1 immunotherapy: a case report. Immunotherapy 12: 1213-1219.

8. Pundole X, Sarangdhar M, Suarez-Almazor ME (2019) Rheumatic and muscoloskeletal adverse events associated with immune checkpoint inhibitors: emerging trends from the US Food and Drug Administration Adverse Event Reporting System. Journal of Immunotherapy and Precision Oncology 2: 65-73.

9. Nicolatou-Galitis O, Kouri M, Papadopoulou E, Vardas E, Galiti D, et al. (2019) Osteonecrosis of the jaw related to non-antiresorptive medications: a systematic review. Supportive Cancer Care 27: 383-394.

10. Ponzetti M, Rucci N (2019) Updates on osteoimmunology: what's new on the cross-talk between bone and immune system. Front Endocrinol 10: 236. 